\title{
Real-Time Compensation of Chatter Vibration in Machine Tools
}

\author{
Dong-Hoon $\mathrm{Kim}^{1} *$, Jun-Yeob Song ${ }^{2}$ \\ ${ }^{1,2}$ Korea Institute of Machinery and Materials, South Korea \\ E-mail: kdh680@kimm.re.kr \\ Suk-Keun $\mathrm{Cha}^{3}$, Han-kyu $\mathrm{Kim}^{4}$ \\ ${ }^{3}$ ACS Co., Ltd., ${ }^{4}$ SMIC Co., Ltd., South Korea \\ E-mail: skcha@acs.co.kr
}

\begin{abstract}
The chatter vibration stands for a sudden relative vibration appeared between a material and a tool while processing with a machine. This vibration is a factor that seriously affects the quality of processed materials as well as being a factor which causes serious damages to the tool and the machine. This study is related to the detection and compensation of chatter vibration that can compensate chatter vibration faster and produce processed goods with more precision by autonomous compensation.

The above-mentioned chatter vibration compensator includes the chatter vibration sensor and the chatter compensator that estimates the compensation value according to the sensor detecting the chatter vibration of mach ine tool and the chatter vibration detected from the sensor while having a feature of being organized by interlocking with the machine tool controller.
\end{abstract}

Index Terms - Machine-tools, Chatter-vibration, Compensation, Embedded-device, $\mathrm{CNC}$

\section{Introduction}

While processing with machine tools, chatter vibration is the thing that has greatest effect on the machining precision next to thermal deformation. Chatter vibration is the relative vibration that is made suddenly between a material and a tool in the processes using machine tool, while this vibration a factor that seriously affects the quality of material to be processed and also act as the reason for causing damages the tool and the machine [1-2].

Accordingly, it is necessary to remove the chatter vibration in order to secure machineability and stability. Chatter vibration created while processing with this machine tool is created under specific conditions. The specification conditions are the case where the varied frequency of cutting ability created while cutting and the resonant frequency of cutter gauge are matching with each other or the case where the variation of cutting ability created while cutting is matching with the fre-quency related to the eccentricity of cutter gauge. In this case, the chatter vibration can be reduced by the control of spindle rate, feed rate and machining depth [3-5].

According to previous studies, chatter vibration can be reduced mainly by the two methods. One is to macroscopically check how much the tool vibrates while at work, and to build a database so that the chatter vibration does not occur again. The other is to collect data with acceleration sensor instead of macroscopic method to analyze the existence of chatter vibration on each processing condition to use the method of selecting the processing condition to prevent creating chatter vibration next time.

However, such methods were not suitable to compensate the chatter vibration on a real time basis to have disadvantage of requiring a lot of time and effort in the compensation of chatter vibration because enough experiment data should be acquired before processing in advance to compensate chatter vibration based on this experiment data. [6-7].

In case the chatter vibration is not properly controlled on a real time basis, the machine tool may break down or the machined material may deteriorate in surface roughness even if it does not break down. In this study, the part on chatter vibration detection and prediction model will be embedded and made into device to be applied by implementing on the CNC (Computed Numerical Controller) which is a controller for functions to control feed rate and speed rate in order to compensate such chatter vibration.

\section{Analysis of Chatter Vibration Signal}

Chatter vibration affects machining accuracy the most next to thermal deformation. Accordingly, it is necessary to remove the chatter vibration in order to secure machineability and stability. Chatter vibration is created in specific conditions. To clarify the conditions that create chatter vibration, acceleration signals were 
measured by the use of the acceleration sensors attached to ' $x$ ', ' $y$ ' and ' $z$ ' axes respectively as shown figure. 1 . Machining measures the acceleration signal of each axis by varying the moving speed, machining depths and spindle rates.

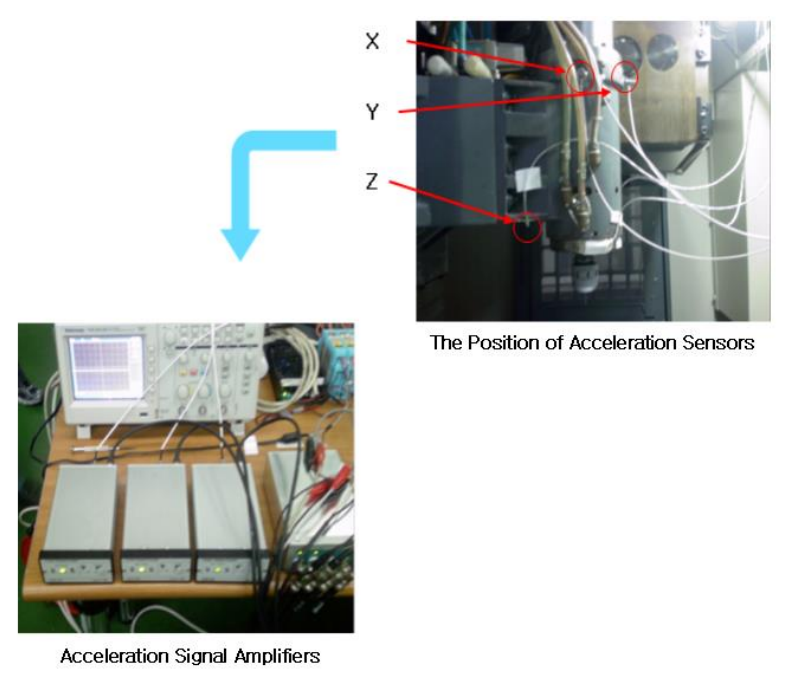

Fig. 1: Basic Environment to Test Chatter Vibration

Machining was done by varying the machining depth, feed rate spindle rate while chatter vibration signals were measured by the use of the acceleration sensors that were magnetically attached to the respective axes of the machine tool. The sampling frequency was determined at $20 \mathrm{KHz}$. Figure 2 shows extracted raw signal and the frequency analysis. The signal was analyzed only in case where the tool was damaged by the excessive chatter vibration created when the spindle rate and the machining depth were fixed but the feed rate is altered. To analyze the characteristics of chatter vibration as shown figure 3, signal FFT (Fast Fourier Transform) was analyzed at the feed rate of 1000, 2000 and $3000 \mathrm{~mm} / \mathrm{min}$ respectively. It was found that the feed rate became higher, as the frequency of amplitude between $0 \sim 500,430 \sim 460$ and $6000 \sim 7000$ became broader. Also, the tool broke down at the feed rate of $3000 \mathrm{~mm} / \mathrm{min}$. To look into the relationship between feed rate and amplitude, the machined surface was examined by the laser sensor. The results are shown in the following figure.

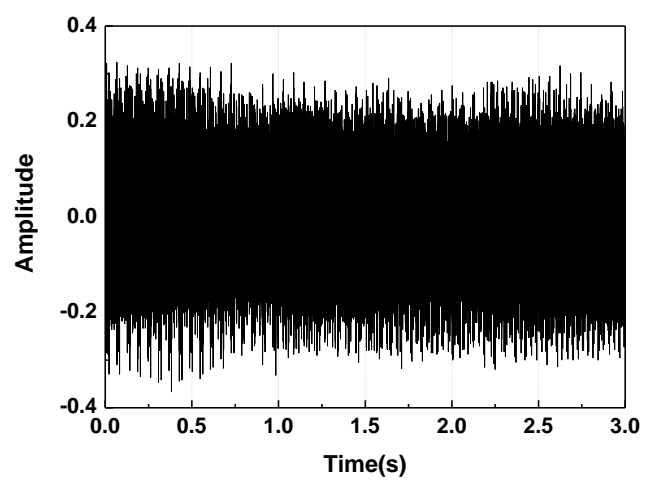

(a) Raw Signal

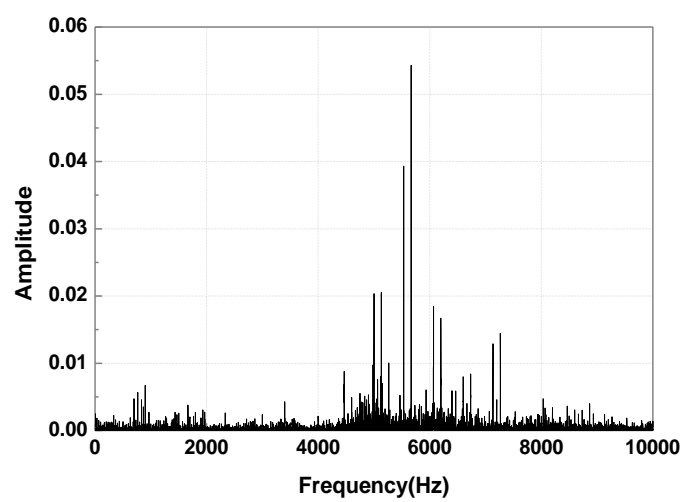

(b) Frequency Analysis

Fig. 2: Machining Signal and FFT (Machining Depth: 0.8mm / Spindle Rate: $500 \mathrm{rpm}$ )

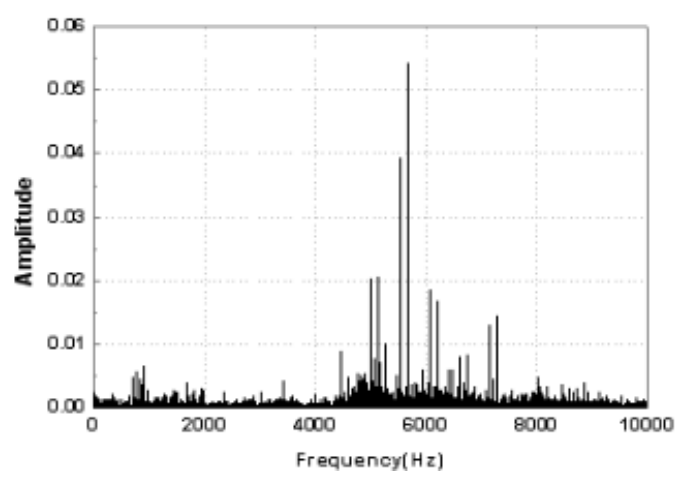

(a) $1000 \mathrm{~mm} / \mathrm{min}$

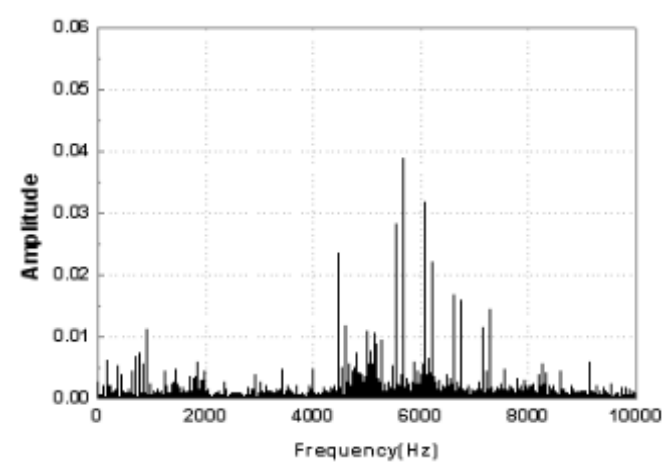

(b) $2000 \mathrm{~mm} / \mathrm{min}$

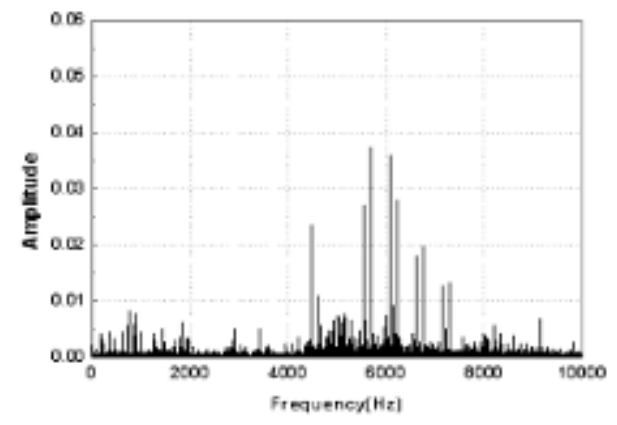

(c) $3000 \mathrm{~mm} / \mathrm{min}$

Fig. 3: Frequency Analysis According to Change of Feed Rate 


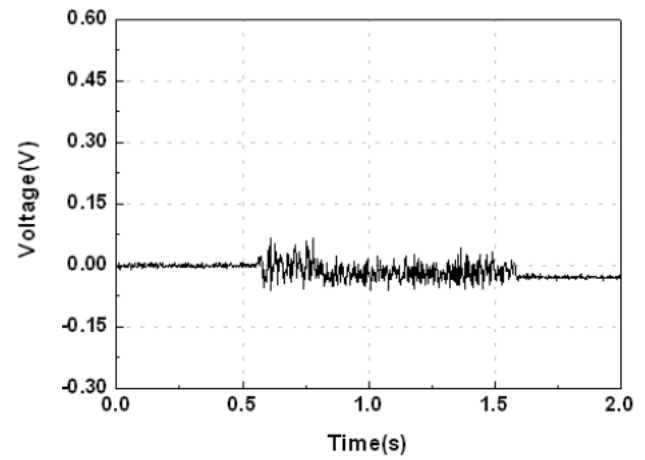

(a) $1000 \mathrm{rpm}, 0.2 \mathrm{~mm}, 500 \mathrm{~mm} / \mathrm{min}$

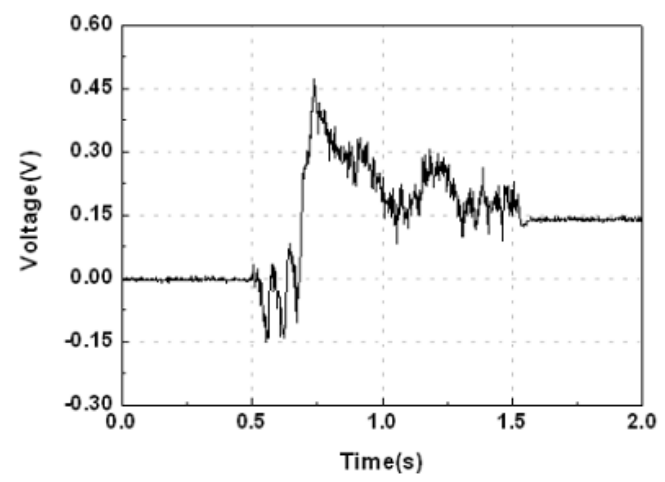

(b) $1000 \mathrm{rpm}, 0.8 \mathrm{~mm}, 500 \mathrm{~mm} / \mathrm{min}$

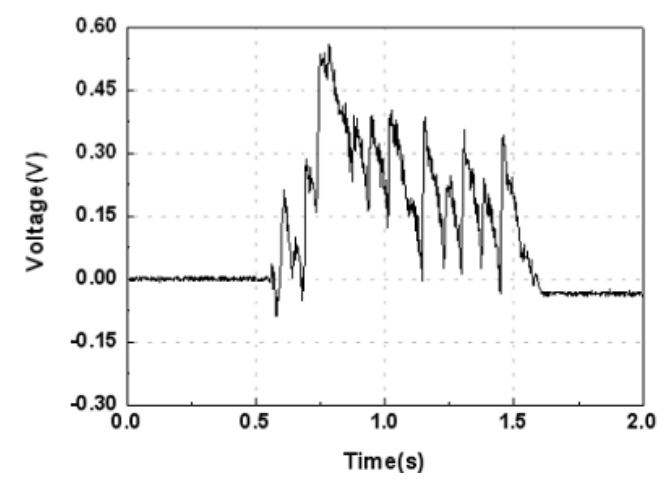

(c) $1000 \mathrm{rpm}, 0.8 \mathrm{~mm}, 1500 \mathrm{~mm} / \mathrm{min}$

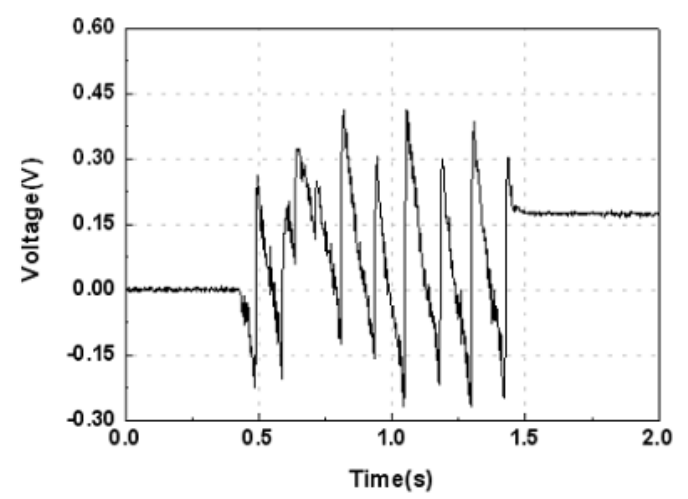

(d) $1000 \mathrm{rpm}, 0.8 \mathrm{~mm}, 2500 \mathrm{~mm} / \mathrm{min}$

Fig. 4: The Alteration of Surface Roughness Related to Machining Conditions
Figure 4(a) shows the surface roughness at the machining depth of $0.2 \mathrm{~mm}$. (b), (c) and (d) show the surface roughness measured at the feed rate of 500 , 1500 and $2500 \mathrm{~mm} / \mathrm{min}$, respectively. Spindle rate and machining depth were $1000 \mathrm{rpm}$ and $0.8 \mathrm{~mm}$ in all cases.

The results show that the surface became rougher as the machining depth became deeper. In addition, the chatter vibration became increased as the feed rate became higher under the same machining condition. In the 3 frequency bands shown in figure 4, the band between 6000 and $7000 \mathrm{~Hz}$ was ideal to distinguish chatter vibrations. Such results imply that real-time data acquisition in the DSP (Dig ital Signal Processor) should be preceded by the band-pass filter.

\section{Embedded Sensing Device and Real-time Compensation}

An embedded device for sensing and determining chatter vibration developed in this study. The device has the size of 175 in length and 80 in width. The Bluetooth shown in the front view is used for the wireless communication with the CNC machine tool. It was designed so that a new order could be communicated from Bluetooth to the CNC machine tool when a chatter vibration is sensed in the compensator. The advantage of such a wireless communication is providing efficiency in build ing client structure if it is necessary to compensate the chatter vibrations that occur simultaneously in many machine tools on a real-time basis. Also, in case of having to build a compensator within poor environment of actual worksite, it is something which always requires attention of workers due to short circuit or destruction of coating of the line connected between machine tool and compensator. And the connecting jack which consists of 9 pins has the function of connecting the power of the DSP in the device to the acceleration sensor signal. Figure 5 shows the schematic diagram of the interaction between the developed device and $\mathrm{CNC}$ machine tools.

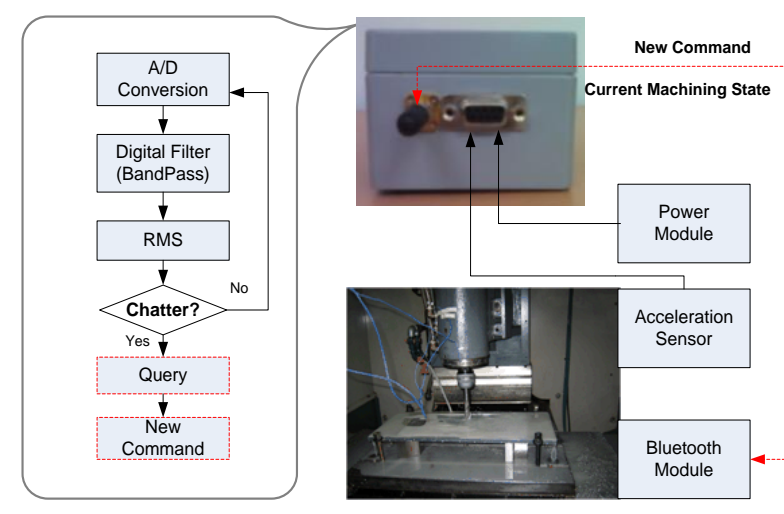

Fig. 5: Interaction between Embedded Device and Machine Tools 


\section{Main Routine}

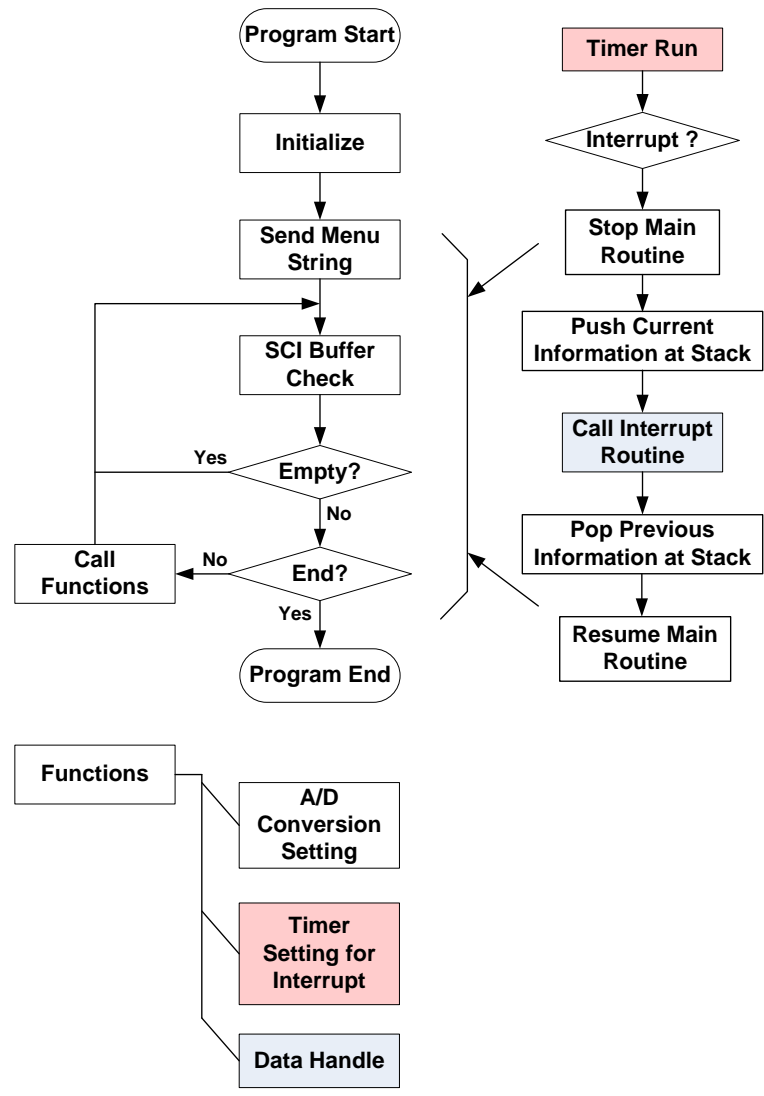

Fig. 6: A Flow Chart of the Main Routine

To sense and determine chatter vibration, a standalone microprocessor was designed and produced to begin with. Then an algorithm determining chatter vibration and a system that can communicate with $\mathrm{NC}$ machine tool in order to reduce chatter vibration have been built. The chatter vibration-sensing and reducing program, developed in line with the purpose of this study, was installed in the embedded device in the form of firmware. The Figure 6 shows the principal structure of the main routine of the program installed in the form of firmware.

\section{$>$ Initialization}

The initialization part consists of a total of 5 types including PLL set-up related to the DSP processing speed, A/D converter set-up to sample the acceleration signal as the reference signal that detects the presence of chatter vibration, SCI (Serial Communication Interface) set-up, internal timer set-up (the reference signal for sampling) and interrupter set-up directly related to sampling. In this study, the sampling frequency was set up so that it can be changed through SCI and the default value was set to $20 \mathrm{KHz}$. The number of A/D conversion channels and the level of input voltage could be adjusted while the signaling speed was fixed at $38400 \mathrm{bps}$.

\section{$>\quad$ Interrupter Process}

A/D sampling is performed in the interrupter routine registered in the initialization. In case an interrupter is created, the main program checks the present condition of $\mathrm{A} / \mathrm{D}$ and if the result of being converted to A/D exists in the buffer, this result is saved to internal variables. When the buffer is saturated after comparing the variable that counts the frequency of interrupter being generated with the buffer size, it deactivates the interrupter-related timer in order to stop interrupters from generating and transmits the character, '>', to the monitoring program being operated in the CNC machine tool. The reason why the interrupter should be stopped is that it takes a longer time to analyze the data in the real buffer compared to the interrupting cycle. To analyze data by the digital filter, RMS (Root Mean Square) and the chatter vibration-determining algorithm, the interrupter must be stopped by deactivating the interrupter-related timer. And after the data analysis process was completed in the main program, the deactivated timer should be reactivated so that the interrupter service routine can be performed. Figure 7 shows this relationship.
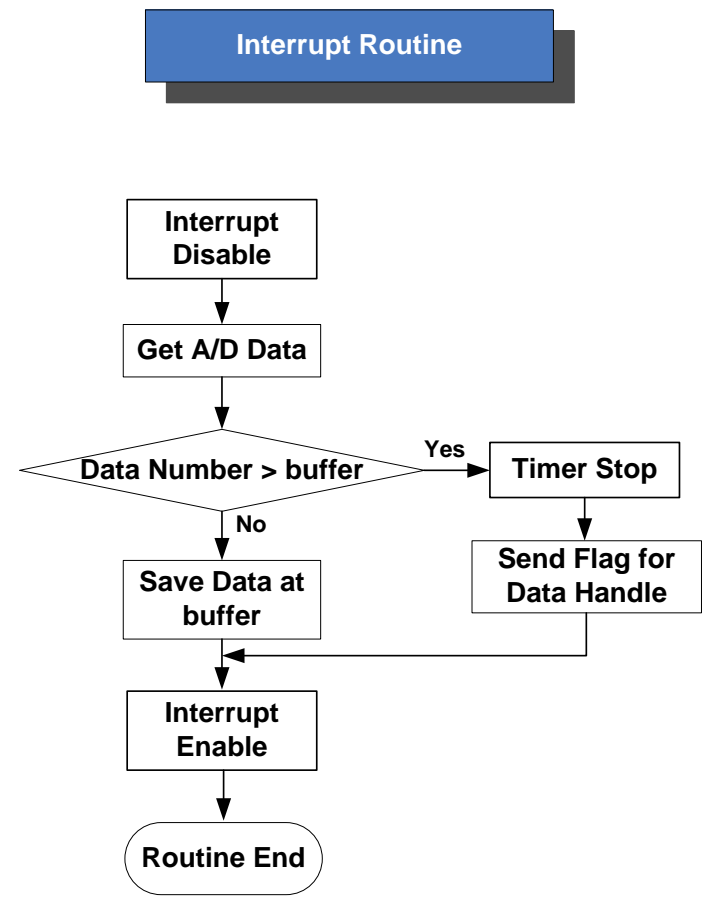

Fig. 7: A Flow Chart of the Interrupt Routine

\section{$>$ The Data Handle Processor}

Figure 8 shows the signal being sampled at high speed to detect chatter vibration and the process of handling this signal. The signal obtained through A/D conversion is saved to the buffer set up in the DSP. Since the capacity of the buffer was not so high, the size of buffer is limited. So the buffer is prevented from overflowing while saving signal on a real time basis and the filtered signals were saved by applying the signal of this buffer to the difference equation which is the designed band-pass filter. And to evaluate the energy 
level of the filtered signal, the root mean square was used as a criterion for detecting chatter vibration to evaluate the energy level of filtered signal. When a sign of chatter vibration was detected, the machining condition is requested to the currently targeted machine tool through serial communication. After the machine tool sends the current machining conditions (feed rate, spindle rate, etc.) to DSP, the new mach ining conditions are ordered to the machine tool based on these conditions. The chatter vibration was compensated by such real-time repeated measurement and the compensation algorithms.

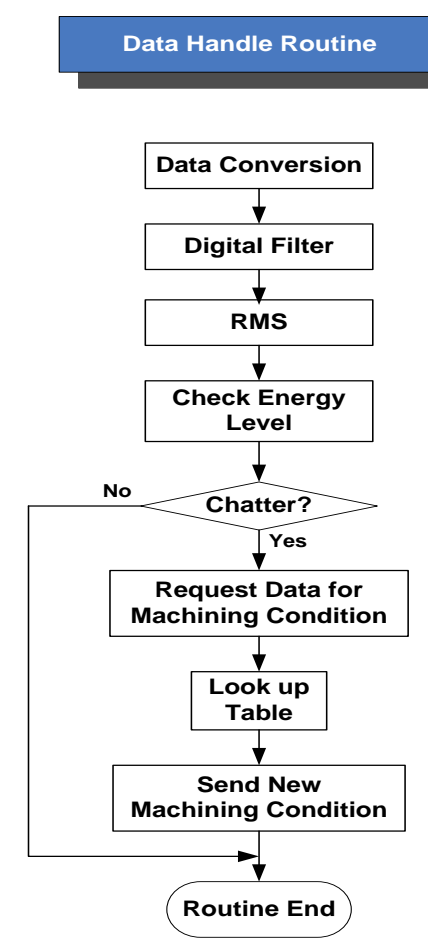

Fig. 8: A Data Handle Flow Chart for Chatter Vibration Compensation
Figure 9 shows the system environment built up to perform on-line real-time CNC-based autonomous compensation. The system consists of the sensor part that obtains the vibration of each spindle, the amplification part that amplifies these signals and removes basic noises, the DSP that obtains signals from the amplification part on a real-time basis, the transmission part that transmits the compensation signals of the DSP to the machine tool and the NC kernel part that implements the compensation signals of the DSP.

The operating mechanism sequence is as follows.

The signals of the acceleration sensor are obtained using the A/D conversion module in the DSP.

A digital algorithm is used to distinguish chatter vibration from the obtained signals.

$>$ The RMS is used to determine the energy level of the filtered signals.

$>$ If the RMS level goes beyond a specific range, it is determined as chatter vibration and the value to compensate this gets transmitted to the RS232 module.

$>$ The RS232 module transmits the received value to the Bluetooth module.

The Bluetooth module transmits data to the receiving apparatus by wireless transmission.

The program which is executed in the machine tool receives this to be analyzed and calls the controllerrelated library to be executed in order to apply the com-pensation value.

The compensation value gets applied and chatter vibration gets reduced.

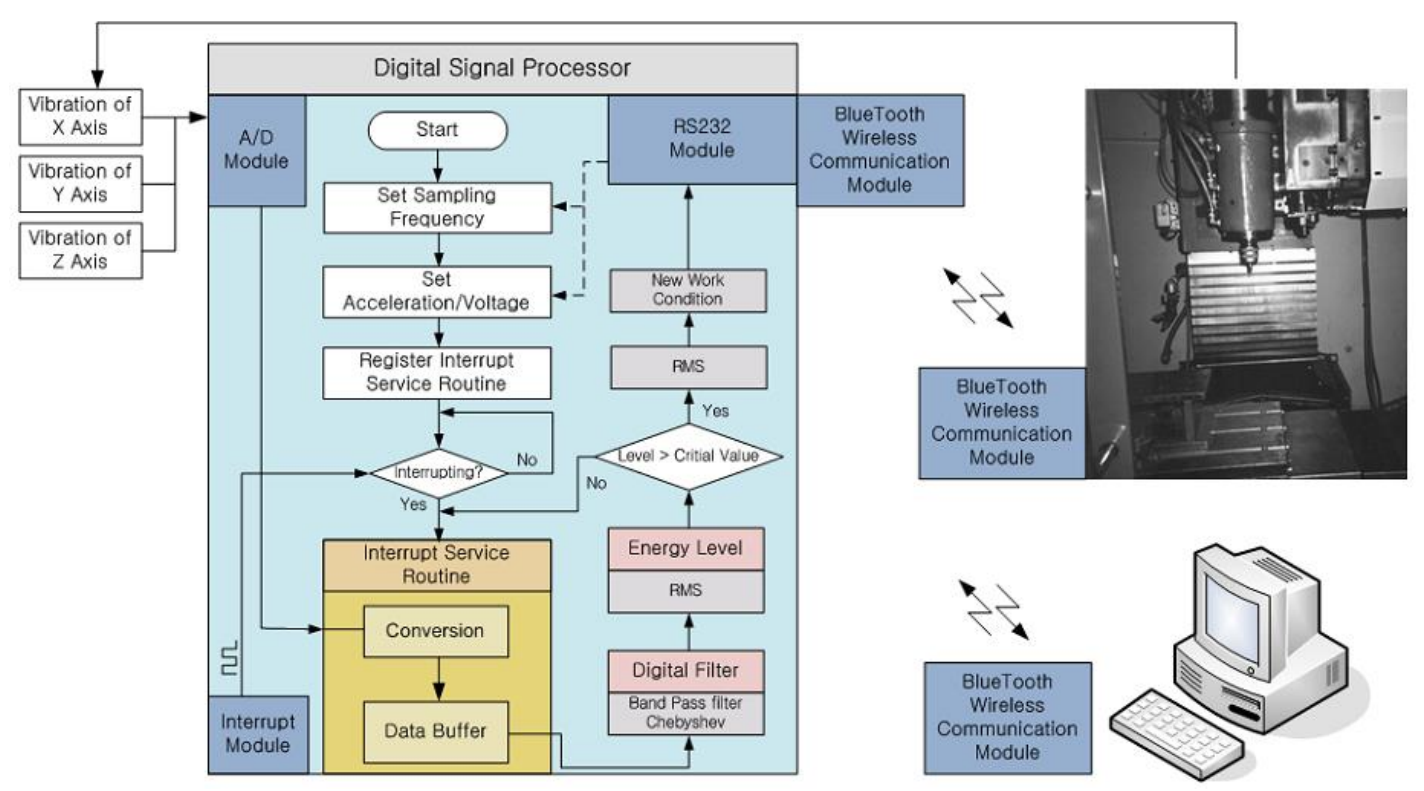

Fig. 9: A Flow Chart of the Real-time Reduction of Cahtter Vibration 
Figure 10 shows the flow for real-time compensation of chatter vibration. To co mpens ate the chatter vibration in the CNC, the ratio of the RMS threshold (created by the chatter vibration that was preceded experimentally) to currently measured RMS value was made as the feed rate variation ratio command of the machine tool to adopt a method of commanding to NC kernel. The experiment conditions are as follows.

\section{Machining Frequency: 5 times}

Spindle Rate: $1000 \mathrm{rpm}$

\section{Feed Rate: $200 \mathrm{~mm} / \mathrm{min}$}

It is considerably difficult to extract measurement data necessary in the process happening on a real-time basis in the DSP. In this study, only the command value of the CNC machine tool was extracted from the DSP. Then, the machined surface was measured by the stylus and was compared to the case where chatter vibration was not compensated. Figure 11 shows the results. The rate of performance improvement was calculated using the following formula.

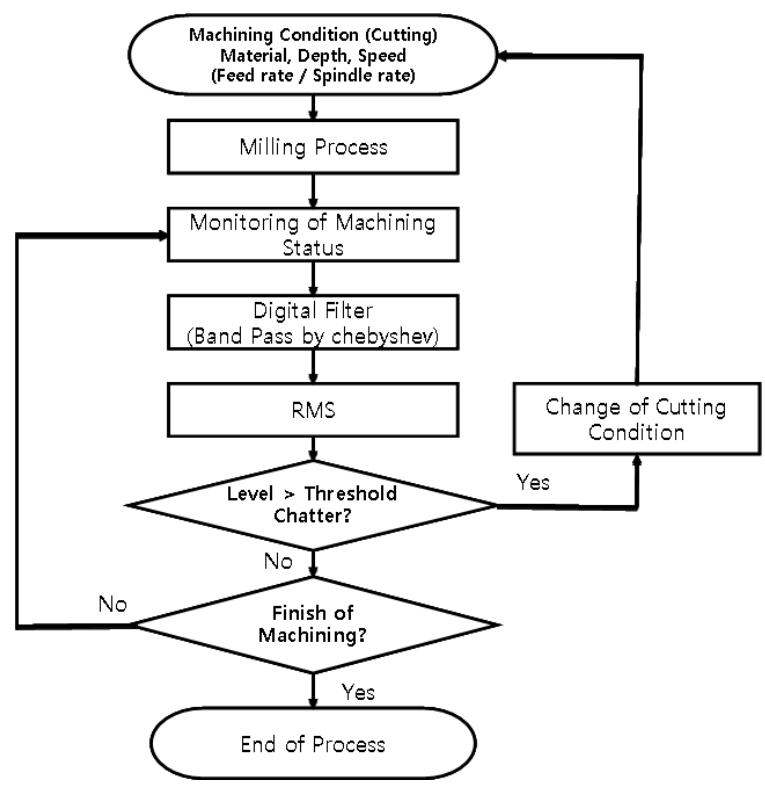

Fig. 10: A Mechanism for Real-time Compensation of Chatter Vibration

$$
\begin{gathered}
\text { Rate of } \\
\text { Performance Improvement }(\%)
\end{gathered}=\frac{\text { Roughness }}{\text { Pre-Compensation }} \begin{gathered}
\text { Roughness } \\
\text { Roughness }
\end{gathered} \times 100
$$

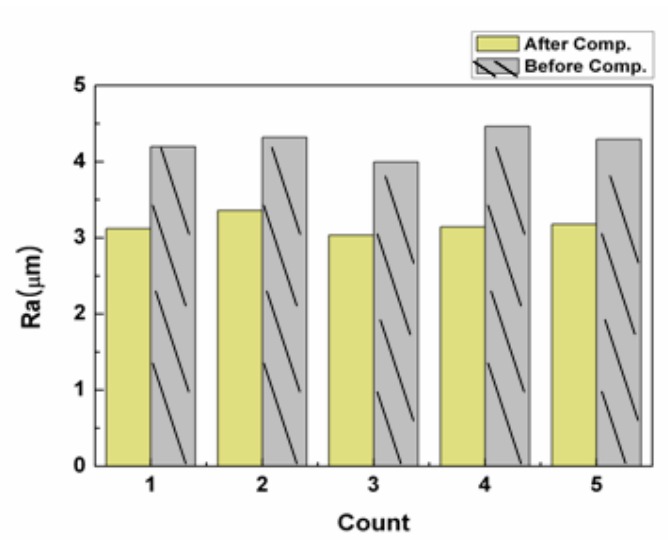

Fig. 11: A Compensation of Surface Roughnesses

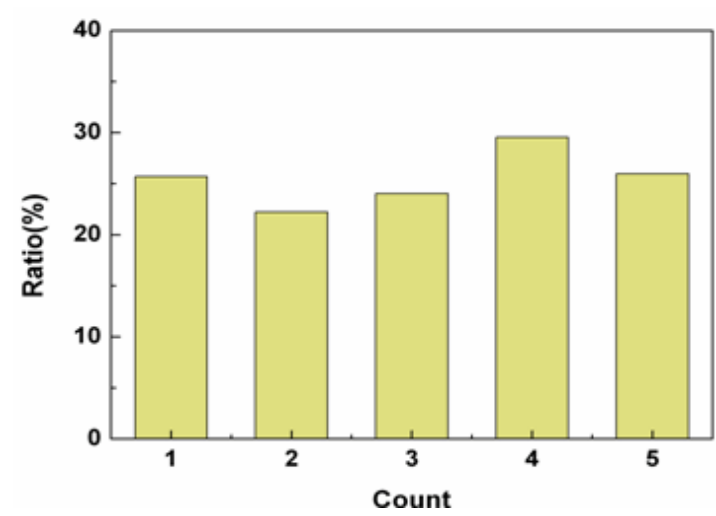

Fig. 12: The Rate of Performance Improvement
The performance improvement rate after chatter vibration compensation through the experiment repeated five times was shown as about $5 \%$. Figure 12 shows the performance improvement rate on each experiment. 'Table 1 ' shows the summary of mean of command values and mean value of surface roughness before and after compensation on the result of repeating the experiment five times

Table 1: The Comparison between Before and After Applying Chatter Vibration Compensation

\begin{tabular}{|c|c|c|}
\hline & $\begin{array}{c}\text { Pre-Compensation } \\
\text { Values } \\
\text { (5 Times Avg.) }\end{array}$ & $\begin{array}{c}\text { Post-Compensation } \\
\text { Values } \\
\text { (5 Times Avg.) }\end{array}$ \\
\hline Feed Rate & $152-200 \mathrm{~mm} / \mathrm{min}$ & $152-200 \mathrm{~mm} / \mathrm{min}$ \\
\hline $\begin{array}{c}\text { Surface } \\
\text { Roughness }\end{array}$ & $4.15 \mathrm{um}$ & $3.11 \mathrm{um}$ \\
\hline
\end{tabular}

\section{Conclusion}

The purpose of this study is to autonomously control the deterioration of surface roughness caused by the chatter vibration made from the spindle rotation and the friction with the material to be machined to be performed according to the following details. A digital filter determining the existence of chatter vibration was implemented after obtaining the signal of machining conditions on a real time basis. Since the acceleration signal is set as the basis through the design of digital 
filter and measurement of energy level through RMS, it was possible to determine the chatter vibration signal while machining on a real-time basis under limitations such as high speed sampling or few nu mber of samples under the environment where automated compensator for compensating chatter vibration is based on microco mputer (DSP) along with high sampling speed. These details were implemented as an embedded device in a form of firmware. The spindle rate, feed rate and machining depth, etc were made to be autonomously compensated on a real-time basis while machining by programming on the interface of $\mathrm{CNC}$ based on these things. The difference in the method of applying compensation is the part which enables change of machining conditions dynamically when chatter vibration is generated and independently developing a program for applying the output compensation value at a compensator in a form of embedded device. In addition, the function of machine tool being controlled by chatter vibration on a real-time basis has been imple mented by transmitting the obtained compensation value from the inner controller of embedded device to the $\mathrm{CNC}$ which is the machine tool controller by wireless communication.

\section{References}

[1] D. H. Kim and Song, J. Y., Ubiquitous-Based Mobile Control and Monitoring of CNC Machines for Development of u-Machine, Journal of Mechanical Science and Technology, 20 (4) (2006) 455-466.

[2] D. H. Kim and J. Y. Song, KnowledgeEvolutionary Intelli-gent Machine-Tool - Part 1: Design of Dialogue Agent based on Standard Platform, Journal of Mechanical Science and Technology, 20 (11) (2006) 1863-1872.

[3] R. Cheah, Design and Implementation of an MMS Environment on ISODE, Computer Communications, 20 (15) (1997) 1354-1364.

[4] D. H. Kim, S. H. Kim and K.S. Koh, CNCimplemented Fault Diagnosis and Web-based Remote Services, Journal of Mechanical Science and Technology, 19 (5) (2005) 1095-1106.

[5] P. K. Wright, Principles of open-architecture manufacturing, Journal of Manufacturing Systems, 14 (3) (1995) 187-202.

[6] I. Yellowley and P. R. Pottier, The integration of process and geometry within an open architecture machine tool control-ler, International Journal of Machine Tools \& Manufacture, 34 (2) (1998) 277 293.

[7] Budak, E. and Altintas Y. "Analytical pred iction of chatter stability in milling - Part1 : General formulation,", Trans. ASME, Journal of Dynamic System, Measurement and Control, (120) (2002)
22-30.

\section{Authors' Profiles}

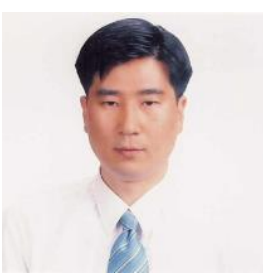

Dong-Hoon Kim received his B.S., M.S. and Ph.D. degrees in Electronic Engineering from Kyungpook National University, Korea, in 1990, 1992 and 2005, respectively. Dr. Kim is currently a principal researcher at the Korea Institute of Machinery and Materials in Daejeon, Korea. His research interests include machine intelligence, M2M and u-Manufacturing.

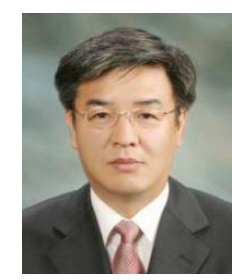

Jun-Yeob Song received his Ph.D. degree in Manufacture Engineering from Pusan National University, Korea, in 2000. Dr. Song is currently a principal researcher and team leader at the Korea Institute of Machinery and Materials in Daejeon, Korea. His research interests include machine intelligence and ultra precision manufacturing.

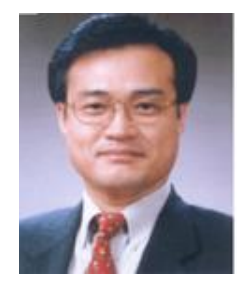

Suk-Keun Cha received the B.S. degree in Electrical Engineering from Mayland University in 1985 . He is currently a Vice President in ACS Co., Ltd, Seoul, Korea. His current research interests include mechatronics, sensors, MES, Middleware, and factory automation

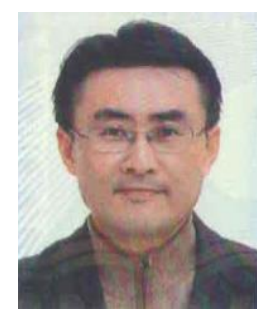

M2M Device.
Han-Kyu Kim received the M.S. degree in Computer Engineering from Wayne State University in 1988. He is currently a President in SMIC Co., Ltd., Seoul, Korea. His current research interests include mechatronics, sensors and actuators, dynamic system modeling, and
How to cite this paper: Dong-Hoon Kim, Jun-Yeob Song, Suk-Keun Cha, Han-kyu Kim,"Real-Time Compensation of Chatter Vibration in Machine Tools", International Journal of Intelligent Systems and Applications(IJISA), vol.5, no.6, pp.34-40, 2013.DOI: 10.5815/ijisa.2013.06.04 